\title{
Filiform-Fire Needle for Upper Extremity Spastic Paralysis After Stroke: A Randomized Controlled Trial
}

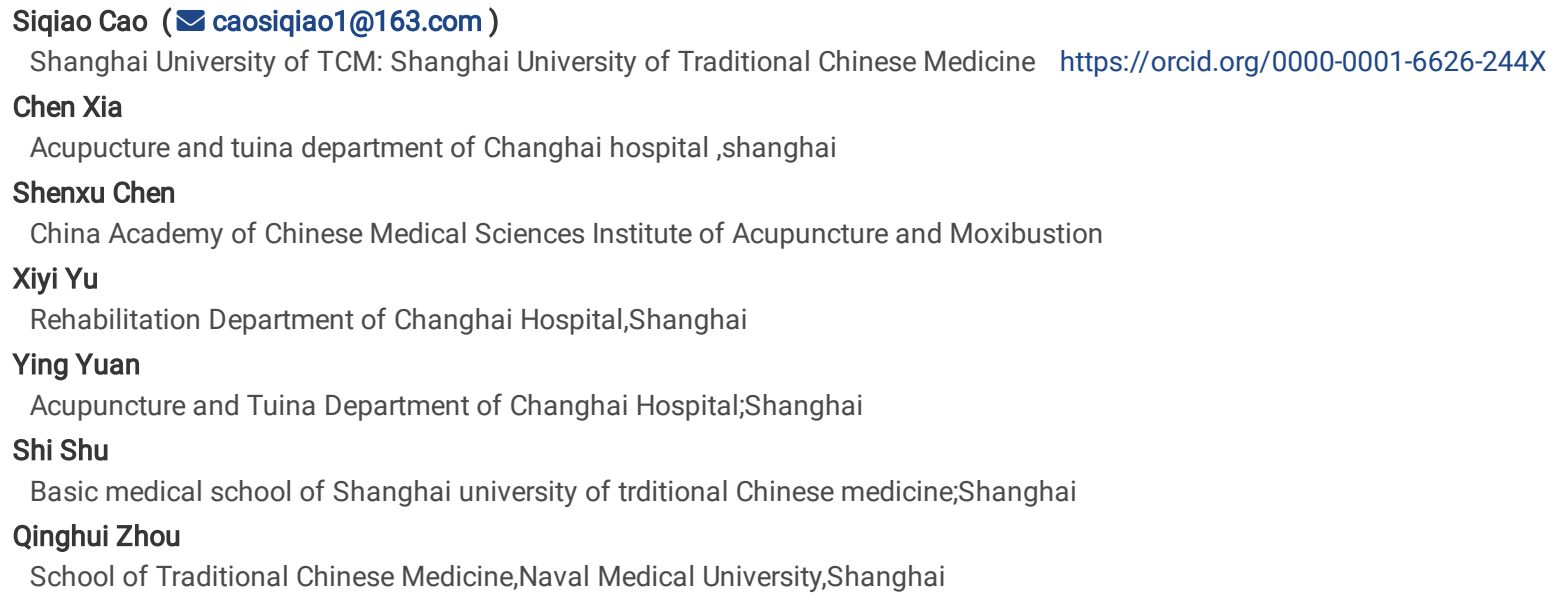

\section{Research}

Keywords: stroke, upper extremity spastic paralysis, rehabilitation therapy, acupuncture therapy, filiform-fire-needle acupuncture therapy

Posted Date: May 20th, 2021

DOI: https://doi.org/10.21203/rs.3.rs-520345/v1

License: (c) (i) This work is licensed under a Creative Commons Attribution 4.0 International License. Read Full License 


\section{Abstract}

Background: Filiform-fire-needle囚FFN囚has been used in clinical treatment of upper extremity spastic paralysis after stroke(UESPAS). This study will evaluate the effect of filiform-fire-needle in the treatment of UESPAS under the background of standardized clinical research, and study the relationship between the efficacy and the course of treatment, so as to provide high-quality evidence-based basis for further clinical treatment and future research design.

Methods: A prospective randomized controlled study was conducted to evaluate the clinical efficacy of FFN acupuncture therapy for patients with UESPAS. UESPAS patients were recruited in Changhai Hospital, Shanghai, China and they were randomly divided into FFN acupuncture group (F group), filiform needle acupuncture group (A group) or rehabilitation treatment group ( $\mathrm{R}$ group). During the 20-day treatment, the same acupoints were taken in $\mathrm{A}$ group and $\mathrm{F}$ group. F group was treated once every 2 days for a total of 10 times, while A group and R group were treated $8 \mathrm{~d}$ in a row and then rest for for 2 days for a total of 16 times. In this study, the primary outcome indicators were modified Ashworth scale (MAS), secondary results including Fugl-Meyer assessment of Upper Extremity Motor Function (FMA-UE), National Institutes of Health Stroke Scale (NIHSS), Barthel index (BI). The above scales were evaluated before treatment, 10 days and 20 days after treatment. The safety was evaluated by self-made acupuncture adverse reaction observation table.

Results: A significant difference was observed in MAS, FMA-UE, BI, NIHSS in the three groups from pre- to post treatment. From the evaluation of the changes of MAS, the curative effect of $\mathrm{F}$ group was better than the other two groups after 10 days of treatment, and $\mathrm{F}$ group was better than the $\mathrm{R}$ group on the 20 th day, but there was no significant difference among the three groups after follow-up. The the difference of FMA-UE score showed F group was higher than the other two groups after 20 days of treatment and follow-up. The change of BI score in group F was higher than that in group R after 10 days of treatment, and the change of $\mathrm{BI}$ score in group $\mathrm{F}$ was higher than that in the other two groups after 20 days of treatment and follow-up. No significant change in the score of NIHSS was found in three groups.

Conclusions: FFN acupuncture therapy has an advantage in relieving upper limb spasm and improving the activities of daily life of stroke patients in the short term, and in the long term, FFN has advantages in improving the motor ability of spastic limbs and improving the ability of daily life of patients.

\section{Introduction}

Spastic Paralysis is the most common complication in patients with hemispheric after stroke, with approximately $90 \%$ incidence in limbs ${ }^{[1-2]}$. Patients with upper extremity spastic paralysis after stroke (UESPAS) are often seen in the clinic with the manifestation of hypermyotonia of upper limb flexor muscle group and abnormal coordination of movement patterns ${ }^{[3-4]}$. Due to their large projection region in the motor region of the cerebral cortex, upper limbs are easily damaged and difficultly repaired. At present, rehabilitation therapies such as anti-spasmodic drugs, traditional medical treatment, physical and occupational therapy is widely used as the main clinical treatment of UESPAS ${ }^{[5]}$. However, some disadvantages like drug toxicity and dependence, high cost resources and professional requirement have limited contemporary rehabilitation therapies widespread in community-level medical service system. Thus, non-pharmaceutical therapies accord with primary Chinese medical conditions to treat UESPAS is critically urged ${ }^{[6]}$.

Meanwhile, Increasing evidence confirmed that acupuncture, as a typical traditional Chinese medicine therapy, had long been widely used to relieve limb spasms after stroke, with significant curative effects and unique advantages ${ }^{[7-9]}$. Fire-needle acupuncture, originating from ancient needling therapies, has been used to treat UESPAP clinically with a recognized curative effect.

Filiform-fire-needle (FFN) therapy is derived from fire-needle acupuncture, but uses filiform-needle as the therapeutic needle. FFN acupuncture therapy combines the advantages of fire-needle and filiform-needle, since the acupoints and possible mechanism of FFN acupuncture therapy are similar to filiform needle therapy, but the techniques differ in needle manipulation and retention time. Compared to the traditional fire-needle ( $0.20 \sim 0.30 \mathrm{~mm}$ in diameter), FFN can replace fire-needle for pricking in clinical practice because of its slim needle body $(0.20 \sim 0.30 \mathrm{~mm}$ in diameter), and it has advantages such as less wound, less pain, and avoiding repeated use.

FFN acupuncture therapy can effectively relieve spastic hemiplegia of stroke, and improve limbs motor function and life quality, achieving a more significant efficacy on spastic paralysis than acupuncture ${ }^{[10]}$. Research showed FFN acupuncture therapy could improve the excitability of brain nerve cells and reduce the apoptosis of glial cells, so as to promote the recovery of motor function after stroke ${ }^{[11-13]}$. Although some studies have investigated FFN acupuncture for spastic paralysis after stroke and yielded satisfactory results, questions still exist in some areas, such as lack of the randomized control method, shortboard of experimental design and nonstandard FFN acupuncture technique ${ }^{[14-16]}$. Therefore, the study aimed to evaluate the clinical efficacy of FFN acupuncture therapy in patients with UESPAS and to explore the relationship between efficacy and course of treatment.

\section{Methods}

\section{The protocol of this study was published in detail during the trial ${ }^{[17]}$}

\section{Study design and participants}

We designed a single-centre, three-armed, randomized, double-blind, controlled trial. Patients with a documented UESPAS were allocated and randomly assigned to three groups (a control group, a Filiform-needle acupuncture group and FFN acupuncture group) through central randomization in a 1:1:1 ratio. This trial was approved by the Ethics Review Committee of Shanghai Changhai Hospital (Approval Document No. Chec2016-107 and Chec2018-073), and was registered by the China Clinical Trial Registration Center (Registration No. ChiCTR - IOR - 17013875). 
All participant were recruited in Department of Rehabilitation, Changhai hospital, and our trial was conducted from December 30,2017 to December 31, 2019. For eligible participants who meet all reuired criteria, it was requested to sign the informed consent before randomization.

Inclusion criteria: $\triangle$ patients meeting the diagnostic criteria of stroke with head CT/MRI confirmed by that cerebrovascular accident occurred in the internal

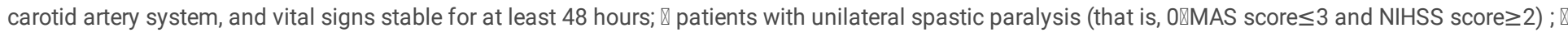
patients with elevated muscle tone, enhanced tendon reflexes, with or without pathological reflexes and with no recent significant change in muscle tension within 2 weeks; $\varangle$ male or females 18-85 years old with the course of 2 weeks to 6 months; $₫$ patients not taking sedatives or muscle relaxants recently; $₫$ patients with informed consent signed.

Exclusion criteria: $\varangle$ patients received other antispasmodic therapy that may affect the observed indicators and efficacy of this study; $\mathbb{Q}$ Pregnant or lactating women, or those with drug allergy history; $\nabla$ patients with cerebral apoplexy for 3 times or bilateral paralysis; $\Downarrow$ patients with increased muscle tension caused by noncerebrovascular disease (e.g., Brain trauma, brain tumors, brain parasitosis or heart disease); $₫$ patients with declined balance and coordination, or patients with cognitive-communication disorders; $\Downarrow$ patients with severe primary diseases(e.g., heart,liver, kidney or hematopoiesis diseases) or mental disease; $₫$ Patients with contraindications in rehabilitation, or filiform-needle, or FFN.

\section{Data collection}

Before treatment, observe and record the basic information of the included patients, such as name, gender, age, height, weight, hemiplegic side, stroke type, ID number, and subject number.All included participants were evaluated upper limb function to complete four scales including Modified Ashworth

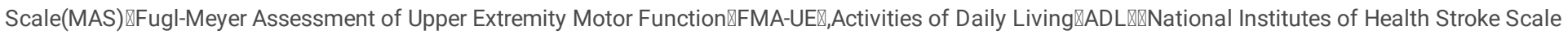
『NIHSS $₫$ before treatment (T0) ,10-day (T1) and 20-day (T2) treatment and the 20th day after treatment (T3).

\section{Randomization and blinding}

Before the study, each patient will be randomly assigned one serial number generated by EXCEL 2013. Assignments were sealed in sequentially-coded and opaque envelopes, and be opened by the researchers following informed consent procedures and baseline testing. Then patients were randomly assigned to three groups according to the order of entry into the study. The assessors and statistician were blinded to the intervention. All therapists were not blinded to group assignments due to the nature of the intervention. Any premature unmasking of a participant would be noted and sent to the researchers.

\section{Interventions}

Patients randomly allocated to three different groups: (1) the Rehabilitation therapy group (R group) as the control group; (2) the Filiform-needle acupuncture group (A group); (3) the FFN acupuncture group (F group). The basic treatment of all enrolled patients was based on Guidelines for Adult Stroke Rehabilitation and Recovery ${ }^{[18]}$.

\section{Rehabilitation treatment group ( $\mathrm{R}$ group)}

Rehabilitation training is formulated according to Guidelines for Adult Stroke Rehabilitation and Recovery of the American Heart Association/American Stroke Association ${ }^{[18]}$. Rehabilitation items of spasmodic paralysis include: physical modalities, postural training, neurodevelopmental therapy, electromyographic biofeedback and so on. The rehabilitation program was carried out 8 days in a row with 2 -day break in 10 days for 20 days (that is, 16 times in total), and every time, the rehabilitation treatment lasted for approximately 30 minutes. All rehabilitation treatments were carried out by qualified professional therapists.

\section{Filiform-needle acupuncture group (A group)}

All participants in this group accepted filiform-needle acupuncture treatment on hemiplegic upper limb with the following acupoints: Jianyu(LI15), Hegu(LI4), Waiguan(SJ5), Quchi(LI11), Shousanli(LI10), Neiguan(PC6), Jiquan(HT1), Chize(LU5), Jianliao(TE-14). Skin was disinfected with 75\% ethanol Before treatment, and disposable stainless steel needles $(0.30 \times 40 \mathrm{~mm}$, Suzhou Medical Appliance Factory, Suzhou, China) penetrate into each acupoint. After getting Deqi, a sense of acid, tingling, distension, and pain, needles should be maintained for 30 min and manipulated every 15 min. Moreover, all the filiformneedle therapy was performed by trained acupuncturists with professional qualification. The acupuncture course was the same as rehabilitation treatment.

\section{Filiform-fire-needle acupuncture group (F group)}

This group will accept FFN acupuncture treatment on the affected upper limb using the same acupoints used in A group. After skin sterilized, disposable stainless steel needles were heated until turning red, and swiftly inserted each acupoint to a depth of 15-25mm, and then pulled out quickly, which lasted less than 0.5 seconds. After each acupoint penetrated, pressure is applied to the insertion site with a alcohol cotton ball. For the acupuncturists performing FFN therapy, they should receive special training to achieve a sound understanding of FFN acupuncture intervention and to normalize the practices across different acupuncturists. The treatment was given every other day in 20 days (that is, 10 times in total).

\section{Abnormal situation of acupuncture and its treatment measures}

If abnormal conditions such as dizzy needles, bleeding and hematoma occur in the course of treatment, the treatment should be stopped immediately, the cause should be found, the degree should be evaluated, and targeted measures should be taken to eliminate the patient's discomfort, as detailed in schedule 1区Schedule 1区. 


\section{Outcomes measures}

The observation period covered the day before treatment $\left(T_{0}\right)$ to 10-day $\left(T_{1}\right)$ and 20-day $\left(T_{2}\right)$ treatment. Follow-ups were conducted at the 20th day after treatment $\left(\mathrm{T}_{3}\right)$.

\section{Primary outcome}

\section{-Modified Ashworth Scale(MAS) ${ }^{[19]}$}

MAS can be used to assess the spasm and resistance of relaxed limbs to rapid passive stretching.In this study,the Modified Ashworth Scale is used to grade

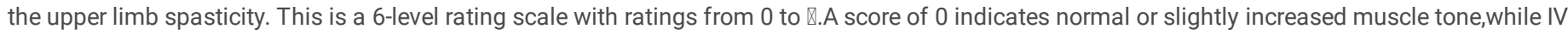
indicates stiff limbs unable to move.

Refer to the "Guiding Principles for Clinical Research of New Chinese Medicines (Trial Version)" published by the Ministry of Health in 2002, and evaluate the efficacy according to the changes in the MAS rating, as follows (Table 1):

\section{Secondary outcomes}

\section{-Fugl-Meyer Assessment of Upper Extremity Motor Function(FMA-UE) ${ }^{[20]}$}

FMA-UR is the earliest and most widely used quantitative assessment scale for stroke motor function in the world, with high reliability and validity. It is divided into upper and lower limbs. The FMA-UE used in this study is the upper limb motor function part of FMA, including 10 major items and 33 small items, with a full score of 66 points. Evaluate the recovery of upper limb function from the aspects of fine movement, tendon reflex, coordination ability. The lower the total score, the worse the upper limb function.

\section{-Activity of Daily Living Scale (ADL) $)^{[21]}$}

ADL consists of the Physical Self-maintenance Scale and the instrument activity of Daily Living Scale, which are used to assess participants' ability to perform tasks of daily living. ADL includes 14 main items, with a maximum score of 64 . The evaluation results can be analyzed according to the total score, scale score and individual items. The total score is less than 16 points as completely normal, and the total score is greater than 16 points as functional decline. This study used the Barthel Index $\mathrm{QB} \otimes$ scale for evaluation, which is very important for evaluating the disability level of patients, assisting in the formulation of rehabilitation plans, and quantifying functional changes.

\section{-National Institutes of Health Stroke Scale(NIHSS) [22]}

NIHSS can comprehensively assess stroke symptoms, and is mainly used for disease judgment, plan formulation, efficacy evaluation and outcome prediction, including 13 meaningful items selected from the Toronto Stroke Scale, the Oxbury Initial Severity Scale and the Cincinnati Stroke Scale. The scale evaluates patients with 13 items in 11 aspects including level of consciousness, gaze, visual field, facial paralysis, limb movement, ataxia, sensation, speech, and neglect. Divided into three levels: light, medium and heavy (light = 0-4 points; medium: = 5-10 points; heavy 11 points)

\section{Safety evaluation}

A self-made abnormal observation table was used (Schedule 1). After each treatment, if adverse events or adverse reactions occur during the trial, the category, degree, duration, treatment measures, treatment results, etc., should be recorded in detail, and on the basis of comprehensive consideration of complications and combined use of drugs, evaluate its correlation with treatment measures. In addition, when adverse events and adverse reactions are found, the person in charge of the study can determine whether to stop the trial according to the condition, and follow up the cases that stop treatment due to adverse events and adverse reactions.

\section{Statistical analysis}

A sample size calculation was determined based on our previous study, in which the effective rates were $83.3 \%, 60.0 \%$ and $50.0 \%$. To detect a significant difference between any two groups with a $80 \%$-power of $80 \%$ and a two-side $5 \%$ significance, these relevant parameters $(83.3 \%, 60.0 \%, 50.0 \%$; $\alpha=0.05,1-\beta=$ 0.9) were input into NCSS-PASS 11, and the calculated number of patients was about 36 per treatment group. Considering an estimated dropout rate of $20 \%$, the total sample size needs 129 initial patients with 60 per group.

SPSS 21.0 software was used to analyze the collected data. Data are expressed as mean \pm standard deviation $\pm \mathrm{s}$ ) or median (interval). Analysis of variance, chi-square test,and rank sum test were used to test the balance of the general information of the three groups of patients (including age, gender, BMl, etc $\square$ ) . Generalized estimation equations or repeated measurement data analysis of variance were used to compare the scores of each scale between timepoints.Data were tested for normality and variance homogeneity before further analysis. $\mathrm{P}<0.05$ was considered to indicate a statistically significant difference.The statistical analysis of data is done by professionals independently who are not participate in the recruitment and treatment of patients, and the researchers and operators work.

\section{Results}


The study was carried out from December, 2017 to December, 2019 in Changhai Hospital in Shanghai. The flow of participants through the trial is shown in Figure 1. Of the 133 potential screened participants, 7 cases were dropped, 7 cases were lost to follow-up. A total of 119 participants were included; 37 participants were allocated to the FFN acupuncture group (F group); 39 participants were allocated to the Filiform-needle acupuncture group (A group).Another39 participants were allocated to the Rehabilitation therapy group (R group) as the control group.

\section{-Analysis of baseline data}

The basic characteristics of participants are summarized in Table 2. There were no significant differences in age, Gender,BIM, stroke types, hemiplegic sides, and the since stroke duration ( $P>0.05$ ) among the three groups of group $\mathrm{R}$, group A and group F. Table 3 showed that no significant differences were found in MAS, FMA-UE,ADLهBI and NIHSS on affected upper extremities $(P>0.05)$ between these three groups prior to intervention.

\section{Primary outcome}

\section{Modified Ashworth Scale(MAS),}

According to the changes in the efficacy of the MAS (Table 4), the total effective rates of patients in the F group, A group and R group for 10 days were $56.76 \%$ (21/37), 30.77\% (12/39), and 30.23\% $13 / 43 \bigotimes$,respectively. the total effective rates of 20 days of treatment were $91.89 \%$ (34/37), 82.05\% (32/39), $67.44 \%$ (29/43); the total follow-up The effective rates were $91.89 \%$ (34/37), 87.18\% (34/39), and 88.37\% (38/43). The efficacy of each group using generalized estimating equations to analyze the results between the two groups: Waldc $^{2}=9.663, \mathrm{P}=0.008$, statistically significant differences between groups. Significantly, the effect of $F$ group is better than the R group (Waldc $\left.{ }^{2}=9.491, P=0.002\right)$. Comparison of repeated measurement time:

Waldc ${ }^{2}=97.090, P=0.001$, the time difference is statistically significant. Compared with follow-up, the probability of treatment for 10 -day and 20 -day is 0.000 and 0.000 , indicating that as the treatment time increases, the effect of treatment is getting better. At the same time, the efficacy of each time point was analyzed by Ridit: On 10-day treatment $\left(\mathrm{T}_{1}\right)$, the efficacy of the $\mathrm{F}$ group was apparently better than $A$ group $(P=0.017)$ and $R$ group $(P=0.010)$;On 20-day treatment $\left(T_{2}\right)$, the therapeutic effect of the $F$ group was significantly better than $R$ group $(P=0.003)$. On 20th day after treatment $\left(T_{3}\right)$, there was no significant difference in efficacy between the three groups $(\mathrm{P}=\mathbf{0 . 1 3 2}$ ). (Figure 2a) (Figure 2b)

\section{Secondary outcomes}

\section{Fugl-Meyer Assessment of Upper Extremity Motor Function(FMA-UE)}

Table 5 shows changes of FMA-UE scores in the F group, A group and R group(Table 5). The variance analysis of the repeated measures design of the difference at each time point found that the FMA-UE scores of the three groups after 10 days of treatment, 20 days after treatment and follow-up were significantly different from those before treatment $(P<0.01)$ \lt suggested that the three treatment methods are all can significantly improve the upper limb function of patients with spasticity. At the same time, the pairwise comparison of the analysis of variance with repeated measures design was performed at each time point.Before treatment(T0), there was no statistically significant difference in the FMA-UE score changes in the $F$ group, A group and $R$ group $(P=0.150)$, indicating that from In terms of short-term effects, the effects of FFN acupuncture, filiform-needle acupuncture and rehabilitation therapy on improving upper limb function are basically the same.On 20-day treatment(T2) and 20th day after treatment (T3), the FMA-UE score of group F changed more than group $A$ and group $R$, and the difference was statistically significant $(P=0.020, P=0.022 ; P=0.012, P=0.020)$, indicating that from the perspective of long-term effects, FFN acupuncture improves upper limb function better than filiform-needle acupuncture and rehabilitation therapy. (Figure 3a) (Figure 3b)

\section{Activities of Daily Living(ADL) Barthel Index (BI)}

Table 6 shows the changes in the scores of daily living ability in groups F, A and R(Table 6). Analysis of Variance using a repeated measure design for the differences at each time point found that the three groups had significant differences in BI scores on10-day treatment(T1) ,20-day treatment(T2)and 20th day after treatment (T3) compared with before treatment ( $\mathrm{Q} \otimes 0.01)$ \lt suggested that all three treatments can significantly improve the daily living ability of patients with spasm. On 20-day treatment(T2) and 20th day after treatment (T3), the change of BI score in group F was greater than that in group A and group $R$, and the difference was statistically significant $(P=0.031, P=0.001 ; P=0.031, P=0.001)$. It shows that the effect of $F F N$ acupuncture on improving daily life ability is better than filiform-needle acupuncture and rehabilitation therapy. (Figure 4a) (Figure 4b)

\section{-National Institutes of Health Stroke Scale(NIHSS)}

Table 7 shows the improvement of neurological deficits in groups F, A and R.Based on the NHISS scores before and after treatment's Generalized Estimating Equations analysis, there was no significant difference among three groups (Waldc2 $=3.601, \mathrm{P}=0.165$ ) (Table 7). It indicated that there was no significant difference in NHISS scores between the three groups.

The estimated marginal mean was: F Group 5.48, $₫$ Group 5.34, R Group 6.55. Repeat measurement time comparison $\varangle \mathrm{Waldc2}=348.177 \varangle \mathrm{P}=0.000 \otimes 0.01 \rrbracket$ the

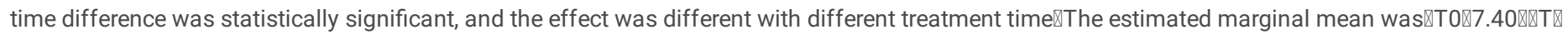

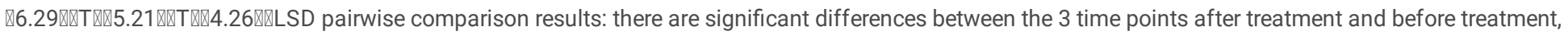
and the difference between before treatment(T0) and 20th day after treatment (T3) is significant. It shows that the three therapies have repairing effects on neurological deficits, and the longer the treatment time, the longer the curative effect and there is no difference in curative effect of the three therapies. ( Figure 5)

\section{Analysis of safety evaluation results}


During the treatment, there were no common adverse reactions such as hematoma, skin infection, bent needle and needle stagnation in the three groups. During the treatment, there were 7 cases of slight bleeding and 6 cases of mild pain in the $F$ group, 9 cases of mild bleeding and 3 cases of mild pain in the A group, and 11 cases of mild pain in the R group, all of which were in the tolerable range. Can continue to receive treatment. In addition, there were 2 cases of moderate pain in the F group, 1 case of faintng during acupuncture treatment and 2 cases of moderate pain in the $A$ group, and a total of 5 patients could not stand it, so they stopped the treatment and withdrew from the trial.

\section{Discussion}

Spastic paralysis stage is recognized as the key period of rehabilitation treatment in the stroke sequela, and during this period, how to induce the generation of separation movement to relieve spasm is the difficulty of improving rehabilitation efficacy and quality of life ${ }^{[2324]}$. FFN are widely used among the patients of stroke sequelae in the clinical. In this study, a randomized controlled clinical study was used to evaluate the difference between FFN, filiformneedle acupuncture and rehabilitation therapy in improving upper limb spasticity, and the improved Ashworth Scale (MAS) Fugl-Myer Upper limb Motor Function Assessment Scale (FMA-UE) Barthel Index (BI) and other scales were used to evaluate, in the hope of developing a safe, effective and economic treatment plan.

In this study, the spasticity was evaluated based on the MAS. Overall, the three treatments can improve the spasm of the affected limb, and with the extension of treatment time, the effect is more and more obvious; among them, the effect of FFN therapy is better than that of rehabilitation treatment, but there is no obvious advantage compared with filiform-needle acupuncture. In the experiment, we found that after the end of the first course of treatment ( $\left.T_{1}\right)$, the curative effect of the F group was the best and was significantly better than that of the other two groups; after the second course of treatment ( $\left.T_{2}\right)$, the curative effect of the $\mathrm{F}$ group was still significantly better than that of the R group, but there was no significant difference compared with the A group; through follow-up, it was found that there was no significant difference in the long-term efficacy of the three treatments. It can be seen that the effect of FFN in improving the spasm of the affected limb is faster and the long-term effect is stable. This may be due to the large amount of stimulation of FFN in a single treatment, which directly relieves the pathological changes of local tissue such as edema, hyperemia, exudation, adhesion and contracture through local mechanical and thermocoagulation, thus relieving myogenic contracture ${ }^{[25]}$. And by dilating blood vessels, speeding up circulation, exuberant metabolism, providing more nutrition for spastic tissue to promote its repair ${ }^{[26]}$

According to the FMA-UE evaluation, the effect of FFN therapy in improving the motor function of the affected limb is better than that of the other two treatments, and the long-term effect is good. By comparing MAS and FMA-UE, we found that FFN in the early rapid relief of spasm at the same time, although the upper limb motor function improved, but there was no difference between groups. This may be due to spasms caused by the emergence of common exercise patterns.In this study, BI was used to evaluate the ability of daily living of patients. The results showed that FFN therapy had a quick and longlasting effect on improving the ability of daily living of patients. It may be that the FFN therapy with a large amount of single stimulation can quickly improve the spasticity of the patients' upper limbs, reduce the occurrence of complications, and then improve the patients' ability of daily livingl.The average course of stroke patients selected in this study was $(50.82 \pm 37.56)$ days, and there was no significant difference in NHISS function score among the three treatments. At this time, it is in the critical period of neurological function recovery of cerebral apoplexy ${ }^{[27]}$. Drugs and rehabilitation methods can directly interfere with the regulation of neural plasticity and promote nerve recovery $\left.{ }^{[28} 29\right]$.

\section{Conclusion}

Taken together, the present results demonstrate that FFN acupuncture therapy can relieve the upper limb spasm of patients after stroke, enhance the motor function of the spasmodic limb, improve patient's ability of daily living and prognosis, and effectively repair the neurologic function.In terms of short-term effects, FFN acupuncture therapy has advantages in relieving upper limb spasticity and improving activities of daily living in patients with stroke.In terms of long-term effects, FFN acupuncture therapy has an advantage in improving the motor ability of spastic limbs and improving the ability of daily life of patients.In summary, FFN acupuncture therapy is a safe and effective treatment with simple operation and few side effects and FFN therapy might be a rational strategy for promoting patients with UESPAS,thus benefiting the increasing number of Spastic Paralysis patients worldwide.

\section{Limitation}

In this study, there are too many subjective scales and lack of objective evaluation indexes, so it is necessary to further improve the credibility and objectivity of the research results. Due to the lack of research on the mechanism of FFN acupuncture, which requires laboratory research to further explain the mechanism of hemiplegia limb spasm after stroke, and reveal the target of FFN acupuncture.

\section{Abbreviations}

ADL『Activities of Daily Living

BI冈Barthel Index

BMIषBody Mass Index

FFN囚Filiform-fire-needle

FMA囚Fugl-Meyer Assessment of Motor Function 
FMA-UE囚Fugl-Meyer Assessment of Upper Extremity Motor Function

MAS『Modified Ashworth Scale

NHISS $₫$ National Institutes of Health Stroke Scale

PROM囚Passive Range of Motion

ROM $₫$ Range of Motion

UESPAS『Upper Extremity Spastic Paralysis After Stroke

\section{Declarations}

Acknowledgements囚Not applicable.

Funding $\bigotimes$ This research received a fund from Science and Technology Commission of Shanghai Municipality(17401933900).

Availability of data and materials

The datasets used in the present study are available from the corresponding author on reasonable request.

Author information

Affiliations

Basic medical school of Shanghai university of traditional Chinese medicine; Shanghai, China Siqiao Cao \& Shi Shu

Acupuncture and Tuina Department of Changhai Hospital; Shanghai, China

Chen Xia\& Ying Yuan

Rehabilitation Department of Changhai Hospital; Shanghai, China

Chen Xia \& Xiyi Yu

Acupuncture and Moxibustion Department of Huadong Hospital; Shanghai, China

Shenxu Chen

Rehabilitation Department of Gonghui Hospital; Shanghai, China

Xiyi Yu

\section{School of Traditional Chinese Medicine, Naval Medical University, Shanghai, China}

Qinghui Zhou

Contributions $\rrbracket$

Siqiao Cao wrote the manuscript, Chen Xia and Shi Shu conceived and executed the study, Shenxu Chen, Xiyi Yu and Ying Yuan collected data. Chen Xia performed data management and statistical analysis. Shi Shu and Qinghui Zhou supervised the performance. All authors read and approved the final

manuscript.

Corresponding author囚Correspondence to Shi Shu and Qinghui Zhou.

Ethics declarations

Ethics approval and consent to participate囚This study was approved by the Ethics Review Committee of Shanghai Changhai Hospital (Approval Document No. Chec2018-073), and was registered by the China Clinical Trial Registration Center (Registration No. ChiCTR - IOR - 17013875).Every participant endorsed informed consent voluntarily.

Consent for publication $₫ A$ ll authors have provided consent for publication of the manuscript in the Journal of Chinese Medicine.

Competing interests $\triangle T$ The authors declare no competing interests.

\section{References}


1. Tang EY, Amiesimaka O, Harrison SL, Green E, Price C, Robinson L, et al. Longitudinal effect of stroke on cognition: a systematic review. J Am Heart Assoc. 2018;7(2):e006443.

2. Hamidi V, Wisløff T, Ringerike T, Linnestad KK, Harboe I, Klemp M. Treatment.

3. of patients with. acute stroke in stroke units (with or without early supported.

4. discharge). Norwegian Knowledge Centre for the Health Services (NOKC).

5. No.18-2010. (2010-06) [2018-10-29].

6. Ivanhoe CB, Reistetter TA. Spasticity: the misunderstood part of the upper motor neuron syndrome. Am J Phys Med Rehabil. 2004;83(10 Suppl):3-9.

7. Li S, Francisco GE. New insights into the pathophysiology of post-stroke spasticity. Front Hum Neurosci. 2015;9:192.

8. Chavez LM, Huang SS, Macdonald I, Lin JG, Lee YC, Chen YH. Mechanisms of acupuncture therapy in ischemic stroke rehabilitation: a literature review of basic studies. Int J Mol Sci. 2017;18(11):E2270.

9. Ottawa P, Khadilkar A, Phillips K, Jean N, Lamothe C, Milne S, Sarnecka J. Ottawa panel evidence-based clinical practice guidelines for post-stroke rehabilitation. Top Stroke Rehabil. 2006;13(2):1-269.

10. Cai Y, Zhang CS, Liu S, Wen Z, Zhang AL, Guo X, Lu C, Xue CC. Electroacupuncture for poststroke spasticity: a systematic review and meta-analysis. Arch Phys Med Rehabil. 2017;98(12):2578-89.

11. Lim SM, Yoo J, Lee E, Kim HJ, Shin S, Han G, Ahn HS. Acupuncture for spasticity after stroke: a systematic review and meta-analysis of randomized controlled trials. Evid Based Complement Alternat Med, 2015, 2015: 870398.

12. Park SW, Yi SH, Lee JA, Hwang PW, Yoo HC, Kang KS. Acupuncture for the treatment of spasticity after stroke: a meta-analysis of randomized controlled trials. J Altern Complement Med. 2014;20(9):672-82.

13. Ye JS. Observation on the efficacy of filiform fire acupuncture in the treatment of spastic paralysis after stroke. Chinese Manipulation Rehabilitation Medicine. 2015;6(1):36 - 7[Chinese].

14. Rao XD, Liu YF, Hong JB, Huang YS. Effect of Fire-needle Acupuncture on the Somatosensory Evoked Potential of the Affected Limb after Stroke. Shanghai Journal of Acupuncture Moxibustion. 2014;33(6):531-2. [Chinese with abstract in English ..

15. Sun LM, Li Y, Zhou Z, Ni H, Zhang P, Ai MM, Liu BH. The Effect of Fire needle on the Expression of Apoptosis Related-protein in Spinal Cord Injured Model Rats. Journal of Clinical Acupuncture and Moxibustion, 2010, 26(12): 53 - 7[Chinese with abstract in English].

16. Sun LM, Li Y, Zhou Z, Ni H, Zhang P, Ai MM, Liu BH. The Influence of Fire Needle on Cell Apoptosis in Spinal Cord Injured Model Rats. Journal of Clinical Acupuncture and Moxibustion, 2011, 27(1): 62 - 5[Chinese with abstract in English].

17. Wang N, Li ZF. Life quality improvement of spastic hemiplegia of stroke treated with fire-needle: a randomized controlled trial. Zhongguo Zhen Jiu. 2008;35(11):1105-9. [Chinese with abstract in English].

18. Yang ZQ, Yuan HN, Huang WF. Clinical observation of the treatment of fire needle for spastic hemiplegia after stroke. Shenzhen Zhong Xi Yi Jie He Za Zhi. 2017;27(20):60-1. [Chinese with abstract in English].

19. Sheng GY, Zhao QL. Application of fire needle for the treatment of spastic hemiplegia after stroke. Neimenggu Zhong Yi Yao. 2017;36(14):115. [Chinese].

20. Xin-yu Yue,Zhuo-qi Feng,Xi-yi Yu,Jia-min Hu,Xiao-Jing He,Shi Shu.Fire-needle acupuncture for upper limb spastic paralysis after stroke:Study protocol for a randomized controlled trial[J].Journal of Integrative Medicine,2019,17(03):167-172.

21. Winstein CJ, Stein J, Arena R, Bates B, Cherney LR, Cramer SC, Deruyter F, Eng JJ, Fisher B, Harvey RL, Lang CE, Mackay-Lyons M, Ottenbacher KJ, Pugh S, Reeves MJ, Richards LG, Stiers W, Zorowitz RD, American Heart Association Stroke Council C O C, Stroke Nursing C O C C, Council on Quality Of Outcomes C R. Guidelines for Adult Stroke Rehabilitation and Recovery: A Guideline for Healthcare Professionals From the American Heart Association/American Stroke Association. Stroke, 2016, 47(6): e98-e169.

22. Griessenauer CJ, Medin C, Maingard J, Chandra RV, Ng W, Brooks DM, et al. Endovascular mechanical thrombectomy in large vessel occlusion ischemic stroke presenting with low NIHSS: a systematic review and meta-analysis. World Neurosurg. 2018;110:263-9.

23. Zhu YH. Rehabilitation Assessment. Shanghai: Shanghai Sciencetific and Techncal Publishers; 2008.

24. Ho J, Ngai SPC, Wu WKK, Hou WK. Association between daily life experience and psychological well-being in people living with nonpsychotic mental disorders: protocol for a systematic review and meta-analysis. Med (Baltim). 2018;97(4):e9733.

25. Lin KC, Hsieh YW, Wu CY, Chen CL, Jang Y, Liu JS. Minimal detectable change and clinically important difference of the Wolf Motor Function Test in stroke patients. Neurorehabil Neural Repair. 2009;23:429-34.

26. Neurorehabilitation group, Chinese Society of Neurology, Chinese Medical Association. Society CS. STROKE SCREENING, PREVENTION AND TREATMENT PROJECT OF MINISTRY OF HEALTH P.CHINA. Guidelines for Stroke Rehabilitation in China (2011 Complete Edition). Chinese Journal of Rehabilitation Theory and Practice, 2012, 18(4): 301 - 18[Chinese].

27. Kucukdeveci AA, Stibrant Sunnerhagen K, Golyk V, Delarque A, Ivanova G, Zampolini M, Kiekens C, Varela Donoso E, Christodoulou N. Evidence-based position paper on Physical and Rehabilitation Medicine professional practice for persons with stroke. The European PRM position (UEMS PRM Section). Eur J Phys Rehabil Med. 2018;54(6):957-70.

28. Hou XY. Scattered Needling with Fire Needles Combined with Anti-Convulsive Rehabilitation in the Treatment of Post-Stroke Spasm of Lower Limbs: Study of Clinical Curative Effect,Motor Functions of Lower Limbs and Walking Function. Journal of Clinical Acupuncture and Moxibustion, 2018, 34(2): $43-6[$ Chinese with abstract in English].

29. Peng YF, Jia ZK, Jiang N. Study on Fire Acupuncture from Yin to Yang in the treatment of Lower limb spasm after Stroke. Cardiovascular Disease Journal of integrated traditional Chinese Western Medicine. 2017;5(30):160-1[Chinese].

Page 8/13 
30. Liu Y, Li CH, Fang YQ, Chen MM, Liao H. Research Progress on Rehabilitation treatment of Stroke. Chin J of Clinical ī1/2²ational Drug Use,2016,9(6):1746[Chinese].

31. Yuan DC, Zhang FC, Wei w, Zheng L. Research progress on neuroplasticity and motor function recovery after cerebral apoplexy. Chinese Journal of Rehabilitation Medicine. 2014;29(4):391-4[Chinese].

32. Shao GF, Jiang JZ. Paying great attention to rehabilitation treatment of nervous system diseases. Chinese Journal of Clinical Rehabilitation. 2003;7(25):3512. [Chinese with title in English ..

\section{Tables}

Table 1 Efficacy evaluation criteria based on MAS changing

\begin{tabular}{|ll|}
\hline Result & Evaluation standard \\
\hline Significant effective & MAS rating restored to 0 or decreased by 2 levels above \\
\hline Effective & MAS rating decreased by 2 levels \\
Improved & MAS rating decreased by 1 level \\
\hline Invalid & MAS rating no change \\
\hline
\end{tabular}

Note: Total effective rate $=$ apparent rate + effective rate + improved rate

Table 2 Comparison of general situation of patients with UESPAS $₫ n=119 \rrbracket \pm s \rrbracket$

\begin{tabular}{|c|c|c|c|c|}
\hline & F group & A group & R group & $\mathrm{P}$ \\
\hline Number of cases $\llbracket n / \% \rrbracket$ & $37 \rrbracket 31.09 \% \rrbracket$ & $39 \rrbracket 32.77 \% \rrbracket$ & $43 \llbracket 36.13 \% \rrbracket$ & \\
\hline Median age $\rrbracket$ range $\mathbb{Z}$ & $70 ه 32-84 \rrbracket$ & 64囚35-84囚 & $65(31-84)$ & 0.182 \\
\hline 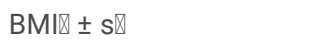 & $24.28 \pm 3.08$ & $23.18 \pm 3.28$ & $24.69 \pm 3.11$ & 0.091 \\
\hline Median duration $₫$ range $\bigotimes$ & 35区14-153】 & $52 \bigotimes 14-172 \rrbracket$ & $32(14-181)$ & 0.199 \\
\hline \multicolumn{5}{|l|}{ Gender冈n/\%区 } \\
\hline Male & $22 \bigotimes 18.49 \% \rrbracket$ & $26 \rrbracket 21.85 \% \rrbracket$ & $30 \otimes 25.21 \% \rrbracket$ & \multirow[t]{2}{*}{0.616} \\
\hline Female & $15 \rrbracket 12.61 \% \rrbracket$ & $13 \rrbracket 10.92 \% \rrbracket$ & $13 \bigotimes 10.92 \% \rrbracket$ & \\
\hline \multicolumn{5}{|l|}{ Stroke types $\ n / \% \bigotimes$} \\
\hline Infarction & $26 \rrbracket 21.85 \% \rrbracket$ & $31 \llbracket 26.05 \% \rrbracket$ & $31 \llbracket 26.05 \% \rrbracket$ & \multirow[t]{2}{*}{0.619} \\
\hline Hemorrhage & $11 \otimes 9.24 \% \rrbracket$ & $8 \llbracket 6.72 \% \rrbracket$ & $12 \bigotimes 10.08 \% \rrbracket$ & \\
\hline \multicolumn{5}{|l|}{ Hemiplegic sides $₫ \mathrm{n} / \% \bigotimes$} \\
\hline Left & $15 \rrbracket 12.61 \% \rrbracket$ & $21 \rrbracket 17.65 \% \rrbracket$ & $22 \llbracket 18.49 \% \rrbracket$ & \multirow[t]{2}{*}{0.471} \\
\hline Right & $22 \rrbracket 18.49 \% \rrbracket$ & $18 \rrbracket 15.13 \% \rrbracket$ & $21 \rrbracket 17.65 \% \rrbracket$ & \\
\hline
\end{tabular}

Note: Rank test was used for age and duration since stroke.One-way analysis of variance was used for BMI.Chi-squared test was used for gender, stroke type and hemiplegic side.

Table 3 Comparison of observational index of patients with UESPAS before treatment $\ n=119 \rrbracket \pm s \rrbracket$

\begin{tabular}{|c|c|c|c|c|}
\hline & F group & A group & $\mathrm{R}$ group & $\mathrm{P}$ \\
\hline Number of cases $\llbracket n / \% \rrbracket$ & $37 \rrbracket 31.09 \% \rrbracket$ & $39 \llbracket 32.77 \% \rrbracket$ & $43 \rrbracket 36.13 \% \rrbracket$ & \\
\hline FMA-UEX $\pm s \rrbracket$ & $29.97 \pm 15.82$ & $29.46 \pm 14.03$ & $26.33 \pm 15.19$ & 0.495 \\
\hline $\mathrm{BI} \otimes \pm \mathrm{s} \rrbracket$ & $49.19 \pm 18.43$ & $49.36 \pm 18.57$ & $46.40 \pm 17.77$ & 0.710 \\
\hline Median NHISS『range囚 & $6 \llbracket 2-17 \rrbracket$ & $6 \rrbracket 1-15 \rrbracket$ & $6 \rrbracket 3-20 \rrbracket$ & 0.333 \\
\hline \multicolumn{5}{|l|}{ MAS $₫ n / \% \rrbracket$} \\
\hline ૫ & $7 \rrbracket 5.88 \% \rrbracket$ & $6 \rrbracket 5.04 \% \rrbracket$ & $7 \rrbracket 5.88 \% \rrbracket$ & \multirow[t]{4}{*}{0.754} \\
\hline$\nabla+$ & $11 \otimes 9.24 \% \bigotimes$ & $10 \otimes 8.40 \% \rrbracket$ & $16 \rrbracket 13.45 \% \rrbracket$ & \\
\hline ( & $15 \rrbracket 12.61 \% \rrbracket$ & $19 ₫ 15.97 \% \rrbracket$ & $15 \rrbracket 12.61 \% \rrbracket$ & \\
\hline ] & $4 \llbracket 3.36 \% \rrbracket$ & $4 \rrbracket 3.36 \% \rrbracket$ & $5 \rrbracket 4.20 \% \rrbracket$ & \\
\hline
\end{tabular}

Page 9/13 
Note: Rank test was used for NHISS.One-way analysis of variance was used for FMA-UE and ADL. Kruskal-Wallis Test was used for MAS. FMA-UE: FuglMeyer Assessment-upper extremity; BI: Barthel Index;NIHSS: National Institutes of Health Stroke Scale;MAS: Modified Ashworth Scale;

Table 4 Changes in efficacy of each group based on MAS®n=119囚

\begin{tabular}{|c|c|c|c|c|c|c|c|c|c|c|c|c|}
\hline \multirow{2}{*}{$\begin{array}{l}\text { Group } \\
\text { 『n/\%区 }\end{array}$} & \multicolumn{4}{|l|}{$\mathrm{T} 1$} & \multicolumn{4}{|l|}{$\mathrm{T} 2$} & \multicolumn{4}{|l|}{ T3 } \\
\hline & $\begin{array}{l}\text { Significant } \\
\text { effect }\end{array}$ & effective & improve & invalid & $\begin{array}{l}\text { Significant } \\
\text { effect }\end{array}$ & effective & improve & invalid & $\begin{array}{l}\text { Significant } \\
\text { effect }\end{array}$ & effective & improve & invalid \\
\hline $\begin{array}{l}F \text { group } \\
\bigotimes n=37 \rrbracket\end{array}$ & 4 & 0 & 17 & 16 & 7 & 5 & 22 & 3 & 11 & 10 & 13 & 3 \\
\hline $\begin{array}{l}\text { A group } \\
\bigotimes n=39 \unrhd\end{array}$ & 1 & 1 & 10 & 27 & 6 & 3 & 23 & 7 & 8 & 8 & 18 & 5 \\
\hline $\begin{array}{l}R \text { group } \\
\bigotimes n=43 \rrbracket\end{array}$ & 1 & 0 & 12 & 30 & 5 & 0 & 24 & 14 & 7 & 7 & 24 & 5 \\
\hline $\begin{array}{l}\text { Waldc2 } \\
\text { هBetween } \\
\text { groups/ } \\
\text { Within- } \\
\text { group》 }\end{array}$ & \multicolumn{12}{|l|}{$9.663 / 97.090$} \\
\hline $\begin{array}{l}\text { P } \\
\text { QBetween } \\
\text { groups/ } \\
\text { Within- } \\
\text { group》 }\end{array}$ & \multicolumn{12}{|l|}{$0.008 / 0.001$} \\
\hline
\end{tabular}

Note: T1 : 10-day treatment T2:20-day treatment. T3: 20th day after treatment

Table 5 Changes of FMA-UE score in each group $₫ n=119 \rrbracket \pm s \rrbracket$

\begin{tabular}{|c|c|c|c|c|c|c|c|}
\hline \multirow[t]{2}{*}{ Group $₫ n \rrbracket$} & \multicolumn{4}{|c|}{ Observing time } & \multicolumn{3}{|c|}{ FMA-UE change value } \\
\hline & T0 & $\mathrm{T} 1$ & $\mathrm{~T} 2$ & T3 & T1- T0 & T2- T0 & T3- T0 \\
\hline F group $\ n=37 \rrbracket$ & $29.97 \pm 15.82$ & $38.03 \pm 15.31$ & $45.43 \pm 14.00$ & $50.08 \pm 11.56$ & $8.05 \pm 4.33$ & $15.46 \pm 6.99 \# *$ & $20.11 \pm 8.18 \# *$ \\
\hline 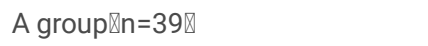 & $29.46 \pm 14.03$ & $36.15 \pm 13.73$ & $41.00 \pm 13.26$ & $45.15 \pm 11.74$ & $6.69 \pm 3.73$ & $11.54 \pm 5.09$ & $15.69 \pm 6.48$ \\
\hline$R$ group $\rrbracket n=43 \rrbracket$ & $26.33 \pm 15.19$ & $32.63 \pm 14.46$ & $38.47 \pm 13.95$ & $42.81 \pm 12.75$ & $6.30 \pm 4.28$ & $12.14 \pm 6.01$ & $16.49 \pm 7.97$ \\
\hline $\begin{array}{l}\text { F『Between groups/ Within-group } \\
\text { /Interaction囚 }\end{array}$ & \multicolumn{4}{|c|}{$1.856 / 468.796 / 3.267$} & \multicolumn{3}{|c|}{$3.993 / 468.796 / 3.267$} \\
\hline $\begin{array}{l}\text { P囚Between groups/ Within-group } \\
\text { /Interaction囚 }\end{array}$ & \multicolumn{4}{|c|}{$0.161 / 0.000 / 0.017$} & \multicolumn{3}{|c|}{$0.021 / 0.000 / 0.017$} \\
\hline
\end{tabular}

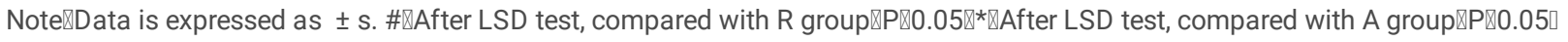

Table 6 Changes of BI score in each group $[n=119 \square \pm s[$

\begin{tabular}{|c|c|c|c|c|c|c|c|}
\hline \multirow[t]{2}{*}{ Group $[n]$} & \multicolumn{4}{|c|}{ Observing time } & \multicolumn{3}{|c|}{ BI change value } \\
\hline & $\mathrm{T}_{0}$ & $\mathrm{~T}_{1}$ & $\mathrm{~T}_{2}$ & $\mathrm{~T}_{3}$ & $T_{1}-T_{0}$ & $T_{2}-T_{0}$ & $T_{3}-T_{0}$ \\
\hline F group $₫ n=37 \rrbracket$ & $49.19 \pm 18.43$ & $57.70 \pm 18.43$ & $66.35 \pm 17.43$ & $74.46 \pm 16.41$ & $8.51 \pm 4.06^{\#}$ & $17.16 \pm 8.38^{\#^{*}}$ & $25.27 \pm 9.20^{\# \star}$ \\
\hline A group $₫ n=39 \rrbracket$ & $49.36 \pm 18.57$ & $56.03 \pm 16.95$ & $62.95 \pm 17.46$ & $70.51 \pm 16.17$ & $6.67 \pm 4.34$ & $13.59 \pm 8.27$ & $21.15 \pm 9.90$ \\
\hline R group $₫ n=43 \rrbracket$ & $46.40 \pm 17.77$ & $51.62 \pm 17.58$ & $58.49 \pm 17.71$ & $66.05 \pm 16.67$ & $5.23 \pm 3.93$ & $12.09 \pm 6.10$ & $19.65 \pm 9.54$ \\
\hline $\begin{array}{l}\text { F囚Between groups/ Within-group } \\
\text { /Interaction囚 }\end{array}$ & \multicolumn{4}{|c|}{$1.428 / 428.031 / 2.710$} & \multicolumn{3}{|c|}{$5.482 / 428.647 / 2.714$} \\
\hline $\begin{array}{l}\text { PQBetween groups/ Within-group } \\
\text { /Interaction囚 }\end{array}$ & \multicolumn{4}{|c|}{$0.244 / 0.000 / 0.038$} & \multicolumn{3}{|c|}{$0.005 / 0.000 / 0.037$} \\
\hline
\end{tabular}

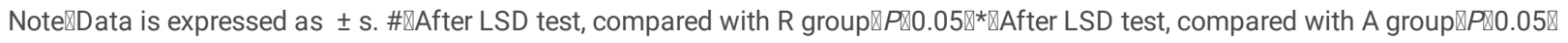

Table 7 Changes of NHISS score in each group $[n=1190$ 


\begin{tabular}{|c|c|c|c|c|}
\hline \multirow[t]{2}{*}{ Group $[n]$} & \multicolumn{4}{|c|}{ Observing time } \\
\hline & $\mathrm{T}_{0}$ & $\mathrm{~T}_{1}$ & $\mathrm{~T}_{2}$ & $\mathrm{~T}_{3}$ \\
\hline F group $₫ n=37 \rrbracket$ & $6 \rrbracket 2-17 \rrbracket$ & $5 \rrbracket 1-16 \rrbracket$ & $4 \rrbracket 1-16 \rrbracket$ & $4 \llbracket 0-13 \rrbracket$ \\
\hline A group $₫ n=39 \rrbracket$ & $6 \rrbracket 1-15 \rrbracket$ & $5 \rrbracket 0-13 \rrbracket$ & $4 \rrbracket 0-11 \rrbracket$ & $4 \llbracket 0-8 \rrbracket$ \\
\hline R group $₫ n=43 \rrbracket$ & $6 \rrbracket 3-20 \rrbracket$ & $6 \rrbracket 2-16 \rrbracket$ & $5 \rrbracket 0-16 \rrbracket$ & $5 \rrbracket 0-15 \rrbracket$ \\
\hline Waldc2邓Between groups/ Within-group $₫$ & \multicolumn{4}{|c|}{$3.601 / 348.117$} \\
\hline P\Between groups/ Within-group $\rrbracket$ & \multicolumn{4}{|c|}{$0.165 / 0.000$} \\
\hline
\end{tabular}

Note $₫$ Data is expressed as $\mathrm{m}$ (range)

Schedule 1 Measures for abnormal situations

Figures

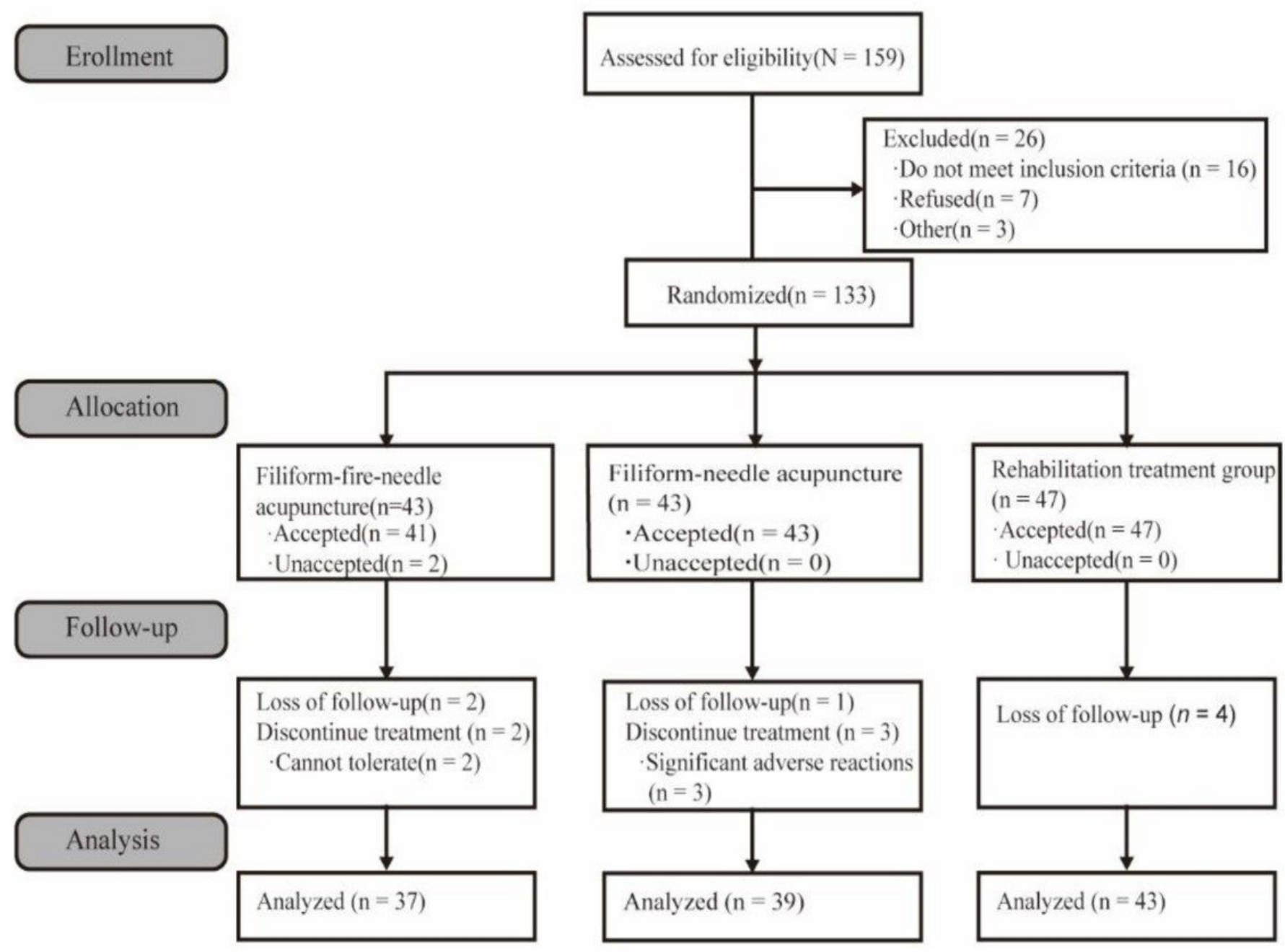

Figure 1

Flowchart of the study design. 

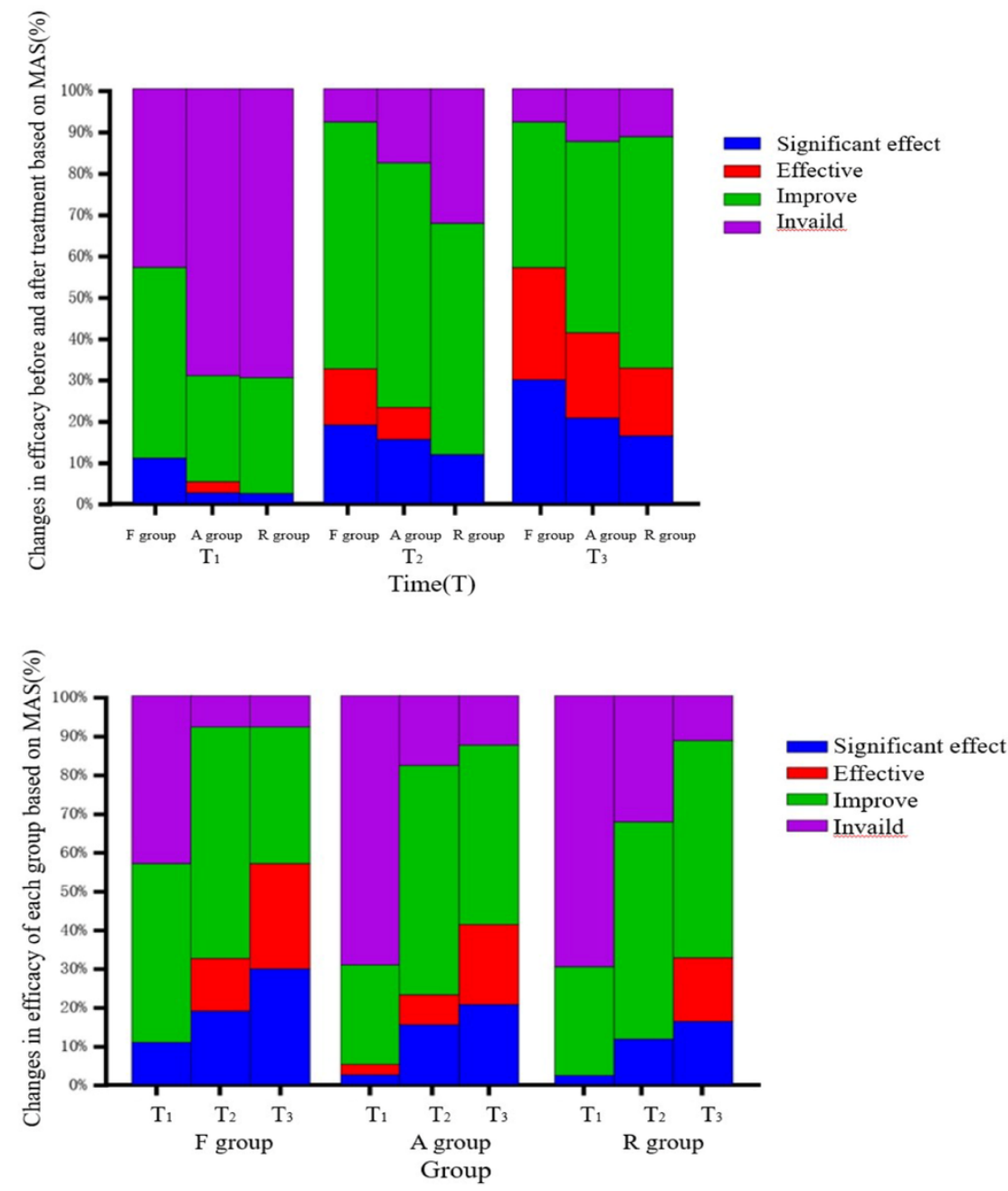

Figure 2

a Changes in efficacy before and after treatment based on MAS Note囚T0:before treatment T1 : 10-day treatment T2:20-day treatment. T3: 20th day after treatment b Changes in efficacy of each group based on MAS Note区T0:before treatment T1: 10-day treatment T2:20-day treatment. T3: 20th day after treatment
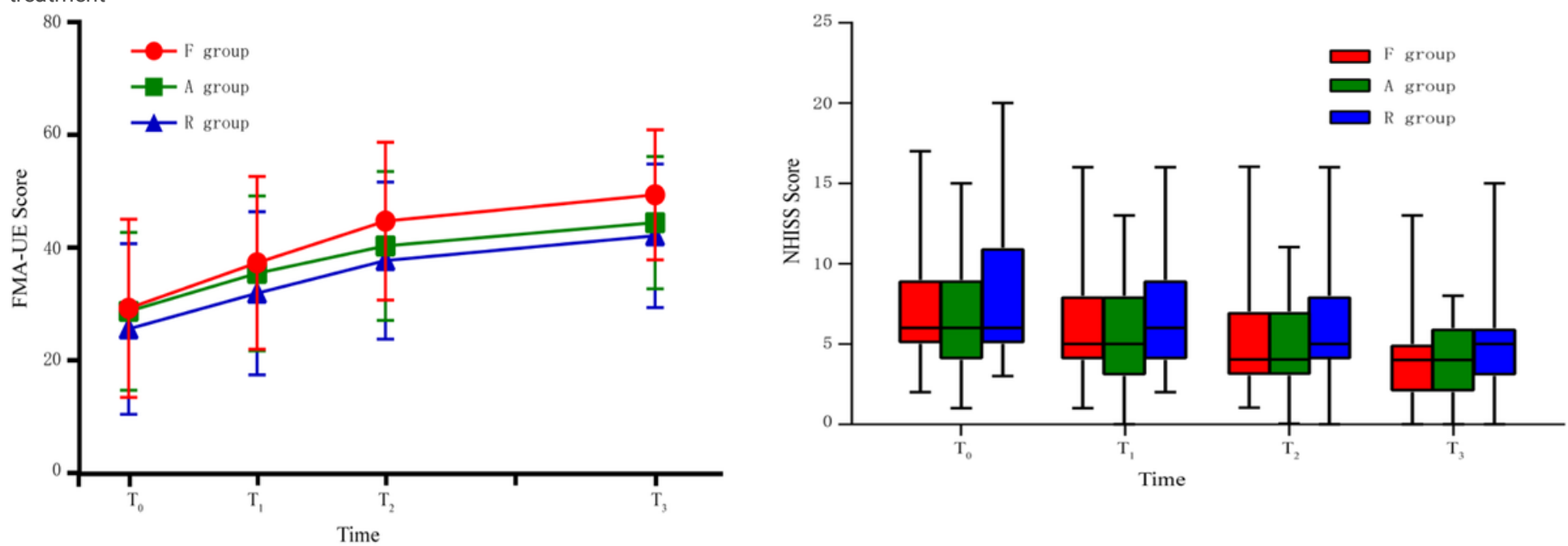

Figure 3

a FMA-UE score trend before and after treatment in each group Note区Data is expressed as $\bar{x} \pm \mathrm{s}$. T0: before treatment T1 : 10-day treatment T2:20-day treatment. T3: 20th day after treatment. b FMA-UE variation trend before and after treatment in each group Note『Data is expressed as $\bar{x} \pm \mathrm{s}$. \#囚After LSD 


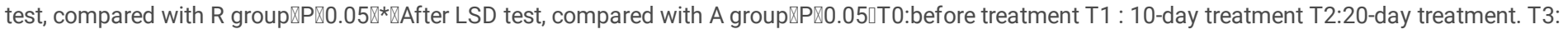
20th day after treatment .
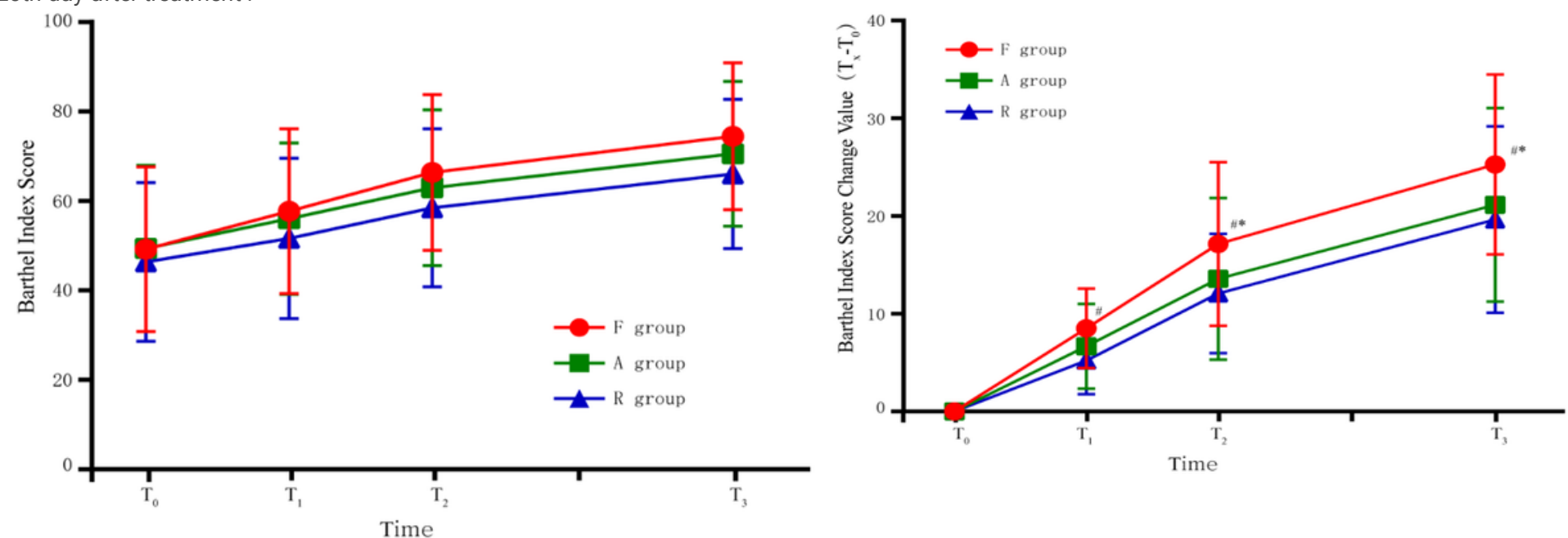

Figure 4

a $\mathrm{BI}$ score trend before and after treatment in each group Note $\triangle \mathrm{D}$ ata is expressed as $\overline{\mathrm{x}} \pm \mathrm{s}$. T0:before treatment T1: 10-day treatment T2:20-day treatment. T3: 20th day after treatment . b BI variation trend before and after treatment in each group Note『Data is expressed as $\bar{x} \pm \mathrm{s}$. \#खAfter LSD test, compared with

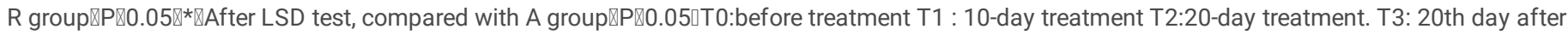

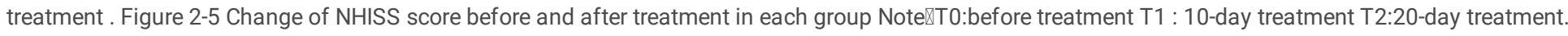
T3: 20th day after treatment

\section{Supplementary Files}

This is a list of supplementary files associated with this preprint. Click to download.

- Supplementary.docx 